\title{
An Empirical Measurement of Signal Attenuation and BER of IEEE 802.15.6 HBC using a phantom solution
}

\author{
Kim Taylor \\ College of Engineering and Science \\ Victoria University \\ Victoria, Australia \\ kim.taylor4@research.vu.edu.au
}

\author{
Daniel Lai \\ College of Engineering and Science \\ Victoria University \\ Victoria, Australia \\ daniel.lai@vu.edu.au
}

\begin{abstract}
The implementation of an IEEE 802.15.6 section 10 compliant human body communications (HBC) transceiver requires an understanding of the signal integrity expected at the receiver. This paper focuses on the reception of IEEE 802.15.6 compliant data packets through the human body. The experiment used a phantom solution to model the electrical characteristics of muscle tissue in a controlled environment. The prototype transmitter was able to achieve a power consumption of $4.5 \mathrm{~nJ}$ per bit using FPGA technology at the minimum spreading factor, while a minimum channel attenuation of $11.4 \mathrm{~dB}$ was measured at $21 \mathrm{MHz}$. Further characterisation of the human body communications channel will be possible with the future development of a portable prototype, optimising the receiver design using the empirical results presented here.
\end{abstract}

\section{Keywords}

Capacitive coupling, Attenuation, IEEE 802.15.6, HBC

\section{INTRODUCTION}

The IEEE 802.15.6 [1] standard was introduced in 2012 to address the need for low power communications over a personal, body area network (BAN). The standard has been divided into three technologies. 802.15.6 sections 8 and 9 cover RF based approaches, using narrow band and ultrawideband modulation schemes respectively. Section 10 describes a baseband communications technique where the transceivers are to be directly coupled to the user's skin. The subject's body tissue forms one half of the communications channel, while the ground connection is completed through the user's environment. This form of coupling is referred to as capacitive coupling in literature due to the lack of DC connection between the transmitter and the receiver.

One of the focuses of the standard is low power consumption. Many RF modulation schemes rely on accurate digital to analogue and analogue to digital conversion, combined with complex signal processing blocks, both requiring significant energy resources. For example the OFDM modulation schemes employed in IEEE 802.11 require high speed Fourier transform computation, combined with high speed digital to analogue conversion. IEEE 802.15.6 section 10 on the other hand aims to directly couple a digital logic signal to the body, via a spectrum mask, avoiding the need for digital to analogue conversion. The scheme that has been adopted has been labelled frequency selective digital transmission (FSDT).

The proposed modulation technique consists of 3 parts: A serial to parallel converter, a Walsh code modulator and a frequency spreader. For IEEE 802.15.6 compliance, the chip rate must be fixed at 42 mega chips per second (Mcps) ${ }^{1}$. The frequency spreader applies a frequency shift code (FSC) consisting of an alternating bit pattern XORed with the output stream of the Walsh modulator. Spreading factors nominated by IEEE 802.15 .6 are $64,32,16$ and 8 . By changing the length of the spreading pattern, a constant chip rate results in four possible data rates, $164 \mathrm{Kbps}, 328 \mathrm{Kbps}$, $656 \mathrm{Kbps}$ and $1312 \mathrm{Kbps}$.

Increasing the spreading factor, increases the likelihood of the receiver correctly determining the phase of the input signal and decoding the transmitted message. This comes at the cost of increased power consumption and a slower data rate as demonstrated in this work.

While the IEEE 802.15.6 standard completely describes the transmitter requirements, the receiver implementation is left to the designer. In order for designers to develop low cost, low power receiver implementations, a knowledge of the body channel characteristics are required. While previous studies have provided an insight into the frequency response of the human body channel, it is worthwhile to observe how well the IEEE 802.15.6 modulation scheme addresses the requirements for a low power and low cost body area network.

Previous studies have been made on the characterisation of the human body channel. Choo et al. [2] developed a lumped element model for capacitive coupling, and did empirical testing on a human subject using a battery powered transmitter module connected to a transmitter electrode and a spectrum analyser connected to a receiver electrode. Their results demonstrated high pass filter characteristics of the

\footnotetext{
${ }^{1}$ A chip rate of $42 \mathrm{Mcps}$ corresponds to a fundamental transmission frequency of $21 \mathrm{MHz}$.
} 
human body channel, attributed to the capacitive return path.

Lucev et al. [4] conducted a similar experiment using a human test subject and a sinusoidal excitation source. Their results showed similar high pass characteristics to Choo's study. Interestingly they found that the receiver contact impedance varied approximately linearly from $100 \mathrm{~K} \Omega$ to $100 \Omega$ over the $100 \mathrm{kHz}$ to $100 \mathrm{MHz}$ test frequency range.

As the focus of this paper is IEEE 802.15.6 HBC transceiver implementation, the excitation source will be IEEE 802.15.6 compliant packets. This experiment aims to vary the chip rate, in order to observe changes in attenuation, power consumption and BER at the data link layer. While IEEE 802.15.6 compliance requires a transmission frequency of $21 \mathrm{MHz}$, it is worthwhile measuring the performance of the scheme at other frequencies. The experiment tested transmission frequencies ranging from $300 \mathrm{kHz}$ to $60 \mathrm{MHz}$.

In order to obtain repeatable measurements, the empirical data obtained in this paper utilised a phantom solution in place of a human test subject. The recipe for the phantom solution was based on Hagmann et al. [3].

\section{EXPERIMENTAL SETUP}

The experiment seeks to collect data on: a) transmitter power consumption, b) channel attenuation and c) bit error rate before and after demodulation. The experimental approach for each of these metrics will be discussed separately. Variables to be considered were chip rate, modulation spreading factor and transmitter, receiver separation.

\subsection{Transmitter setup}

A prototype IEEE 802.15.6 compliant transmitter has been implemented using a Xilinx Virtex 5 FPGA device. The transmitter design consists of a modular peripheral written in VHDL that may be interfaced with an embedded CPU. The prototype also implements this CPU on the same FPGA using the Xilinx Microblaze core, in order to create a compact transmitter design, requiring only a single FPGA. Throughout this experiment, the transmitter has been programmed to transmit the longest packet length prescribed by IEEE 802.15 .6 (256 bytes). As required by IEEE 802.15.6, the transmitter includes a pseudo random sequence generator, or scrambler. The output of the scrambler is XORed with the transmit stream, resulting in a pseudo random transmit sequence.

The FPGA device used, a Xilinx Virtex 5, has a maximum logic clock frequency of $400 \mathrm{MHz}$, as such the highest transmission frequency clock requirements $(120 \mathrm{MHz})$ fall well within the device's operating range.

In order to obtain an estimate for power consumption, a gate level simulation of the transmitter logic has been run for each transmission frequency and modulation rate. The results of the simulations provide a profile for the toggle rates of all gates involved in packet transmission. This information is then fed to the Xilinx Xpwr tool, which provides an accurate power estimate for the FPGA device. This method allows for precise measurement of transmitter power consumption, allowing for separation of dynamic power consumption from

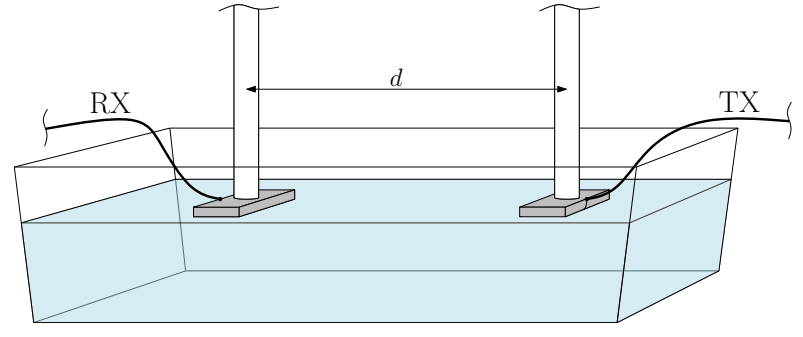

Figure 1: Experiment setup showing electrode placement and fluid. $d$ denotes the distance between the centre of the electrodes.

the FPGA device's static power consumption. ${ }^{2}$ The power consumption results presented in this paper do not include static power consumption.

\subsection{Phantom model setup}

Channel attenuation was measured using a test apparatus consisting of a transmitter electrode, a phantom solution and a receiver electrode. The phantom solutions used were based on recipes from [3] and have been reproduced in Table 1. The solutions have been designed to provide the equivalent conductivity and permittivity of human muscle tissue. In order to approximate the complex environment of muscle tissue with a homogeneous solution, it is necessary to alter the concentrations of salt and glycine for different transmission frequencies. As shown in Figure 1 the values presented are those that are available in literature. The first column of Table 1 matches the nearest transmission frequency to each published solution composition.

The electrodes were placed on top of the solution as shown in Figure 1. The horizontal spacing $d$ between the centre of the transmitter and receiver electrodes was set at either $11.25 \mathrm{~cm}$ or $22.5 \mathrm{~cm}$ from the centre of the electrodes.

The electrodes have two copper layers, isolated by a dielectric. Closed cell rubber was used in order to prevent any of the phantom solution from occupying the space between the ground and signal copper planes. Figure 2 shows the construction of the electrodes. The dimensions were chosen to be similar to a prototype transceiver that is being developed concurrently.

An oscilloscope was connected to both the transmitter and receiver electrodes, allowing for attenuation measurements. A Tektronix MSO5204 10GS/s was used at full sampling rate. The channel 1 and channel 2 inputs were programmed for $50 \Omega$ termination. The oscilloscope has a common ground for all input channels. In practice, wireless devices will not have electrical connection and will rely on capacitive coupling between the ground layer of the electrodes. The experimental setup achieved this by separating the ground electrodes using a balun, and through the use of a battery powered transmitter. Figure 4 is a schematic of the experiment

\footnotetext{
${ }^{2}$ Static power consumption is device dependent and primarily consists of transistor leakage current in the FPGA device. Dynamic power is associated with the transmitter switching activity, and is a function of node capacitance, supply voltage and switching frequency [9].
} 


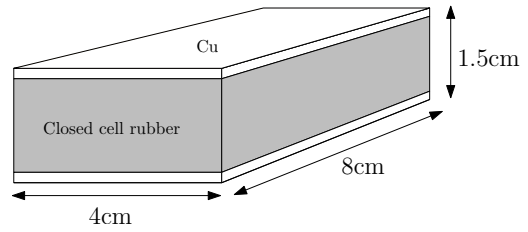

Figure 2: Electrode detail. The dimensions were chosen to approximate the prototype transciever construction.

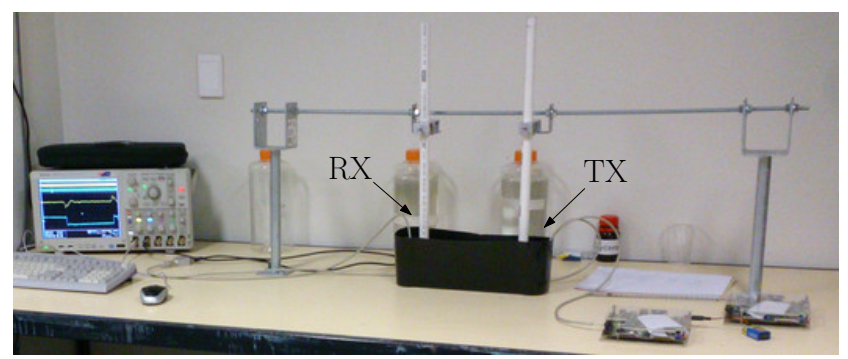

Figure 3: Photograph of experimental setup, showing the transmitter and receiver electrodes suspended in the phantom solution.

setup.

The attenuation information presented here was generated by performing a fast Fourier transform FFT on the oscilloscope PCM sample data, and normalising the results based on the received waveform on channel 2. As shown in Figure 4 , the signal on the receiver electrode is connected to channel 1, while the reference signal is connected to channel 2 . The attenuation was calculated as the difference between the channel 1 and channel 2 FFT coefficients at the frequency of transmission, then averaged using the two adjacent FFT coefficients.

Given the channel 1 raw real valued ${ }^{3}$ PCM samples, $x_{0}, \ldots, x_{N-1}$ and the resultant FFT coefficients, $X_{0}, \ldots, X_{N / 2}$, where the closest coefficient to the transmission frequency is $X_{T}$. The attenuation was calculated as:

${ }^{3}$ As the input data is real valued, $X_{N-k}=X_{k}^{*}$, thus we only require coefficients $X_{0}(\mathrm{DC})$ to $X_{N / 2}$ (the Nyquist frequency).
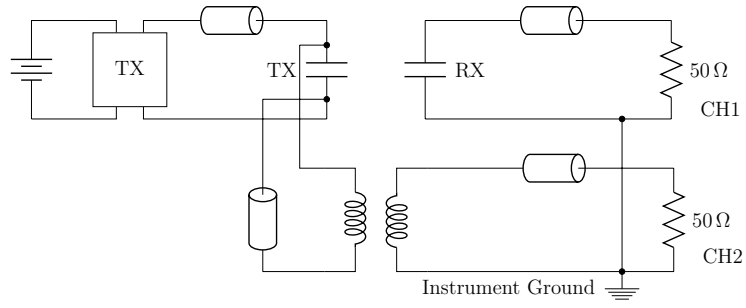

Figure 4: Experiment schematic diagram. The transmission lines shown are $50 \Omega$ cables. Each $50 \Omega$ resistor represents the input stage of the oscilloscope.

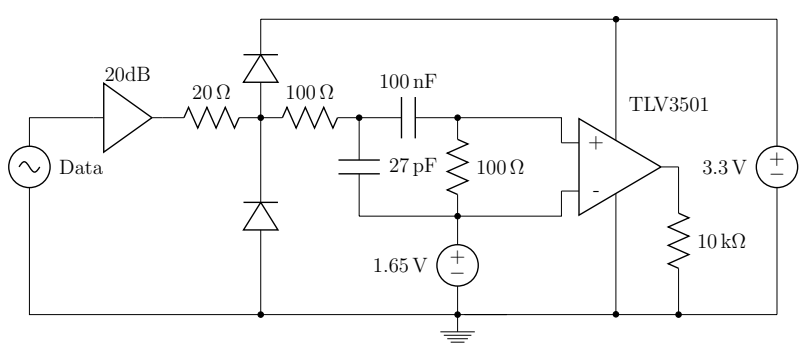

Figure 5: Receiver analogue front end spice model. The 20dB gain block and associated clamping diodes used to model an off the shelf low noise amplifier.

$$
\frac{1}{3}\left[\left(Y_{T-1}^{\prime}+Y_{T}^{\prime}+Y_{T+1}^{\prime}\right)-\left(X_{T-1}^{\prime}+X_{T}^{\prime}+X_{T+1}^{\prime}\right)\right]
$$

where $Y_{0}, \ldots, Y_{N / 2}$ are the FFT coefficients for channel 2, and $X_{n}^{\prime}=20 \log _{10}\left|X_{n}\right|$ and $Y^{\prime}=20 \log _{10}\left|Y_{n}\right|$. The coefficients $X_{n}$ and $Y_{n}$ were normalised such that $\max \left(Y_{n}^{\prime}\right)=0$.

In order to compensate for transmitter to receiver coupling thought the cables and RF propagation from the electrodes, the experiment was repeated with air used in place of the phantom solution. The electrode spacing and position was the same for both the air and fluid measurements.

\subsection{BER measurement}

The received waveform was sampled at $10 \mathrm{Gs} / \mathrm{s}$ and imported into a SPICE model for the receiver analogue front end. The analogue component of the receiver consists of filtering and hard decision detection using a Texas Instruments TLV3501 comparator. The analogue front end schematic is shown in Figure 5. This data was then imported into VHDL test bench for the receiver logic which, like the transmitter, has been implemented on a Xilinx Vertex-5 FPGA device.

The receiver architecture was then simulated and the demodulated data exported. The demodulation procedure includes data recovery, phase alignment, comma detection, packet rate detection, serial to parallel conversion, Walsh code detection based on hamming distance, and descrambling. A graphical depiction of the designed receiver architecture is shown in Figure 6 The demodulated packets were then compared with the transmitted packets in order to calculate BER at the data link layer. In order to demonstrate effectiveness of the Walsh coding scheme for error correction, the received chips after hard decision detection were also compared to the transmitted data and presented as raw BER.

The first stage of the receiver, data recovery, consists of a $4 \mathrm{x}$ oversampling stage and edge detection logic. $4 \mathrm{x}$ oversampling is a result of using four phases of a clock signal set at twice the transmission frequency. The structure of this data recovery block closely follows that presented by Xilinx in their application note 225 [6].

Receiver synchronisation is achieved in the phase alignment stage. An IEEE 802.15.6 packet starts with a preamble of a known sequence repeated 4 times. The receiver checks for 
Table 1: Formulations for muscle simulating phantoms matched to transmission frequencies.

\begin{tabular}{|c|c|c|c|c|c|c|}
\hline \multirow{2}{*}{$\begin{array}{c}\text { Transmission } \\
\text { Frequency } \\
\text { (MHz) }\end{array}$} & \multirow{2}{*}{$\begin{array}{c}\text { Solution } \\
\text { Frequency } \\
(\mathrm{MHz})\end{array}$} & \multicolumn{3}{|c|}{ Weight Percent } & \multicolumn{2}{|c|}{$\begin{array}{l}\text { Dielectric } \\
\text { Properties }\end{array}$} \\
\hline & & Water & Glycine & $\mathrm{NaCl}$ & $\epsilon^{\prime}$ & $\sigma(\mathrm{S} / \mathrm{m})$ \\
\hline $\begin{array}{l}0.300,2.000 \\
5.000,13.00\end{array}$ & 13.56 & 79.4 & 20 & 0.58 & 145 & 0.6 \\
\hline $21.00,30.00$ & 27.12 & 88.5 & 11 & 0.49 & 112 & 0.6 \\
\hline $\begin{array}{c}42.00,55.00 \\
60.00\end{array}$ & 40.68 & 93.5 & 6.0 & 0.52 & 93.5 & 0.7 \\
\hline
\end{tabular}

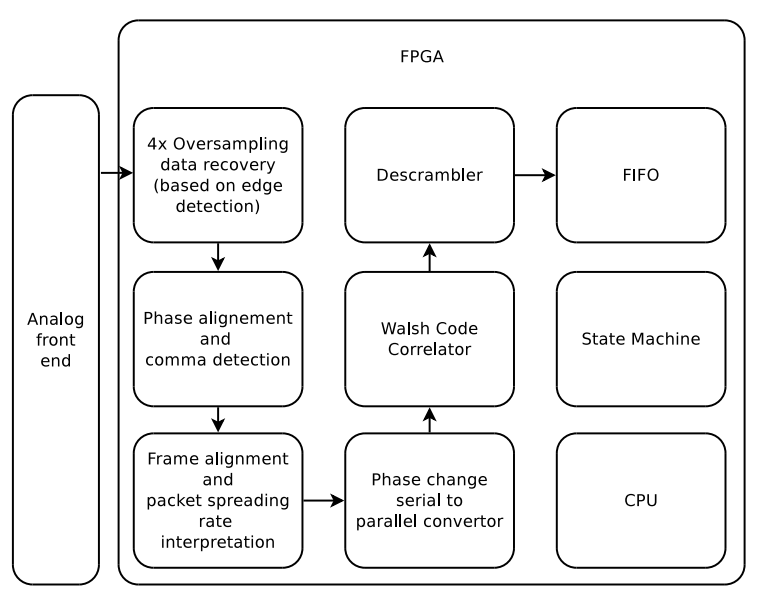

Figure 6: Prototype receiver architecture, as implemented in hardware using a Xilinx Virtex-5 FPGA device. A simulation of this hardware was used in order to allow detailed analysis of operation.

this known sequence by calculating the hamming distance between the input data and the packet preamble. This is done for each possible phase alignment. IEEE 802.15.6 stipulates that a spreading factor of 8 is used for the preamble. Consequently there are 8 possible phase alignments. Once the preamble sequence has been detected, the best phase is selected based on hamming distance and detection of phase transitions on the input stream. An earlier receiver design used detection of phase transitions only, however this was found to be inadequate when phase transitions are distorted by the communications channel. Efficient calculation of hamming distance is achieved using look up tables LUTs based on the methods published by Sklyarov et al. [7].

Once the receiver has been synchronised, the VHDL test bench then prints both the raw data stream and the demodulated data to file for comparison with the original transmitted message.

\section{RESULTS AND DISCUSSION}

\subsection{Transmitter power consumption}

The results of the Xilinx Xpwr simulations for the transmitter hardware are presented in Figure 7. The maximum variation in power consumption for each spreading factor was less than $2 \%$.

This result is consistent with the modulation scheme's implementation of spreading factor. Doubling the spreading

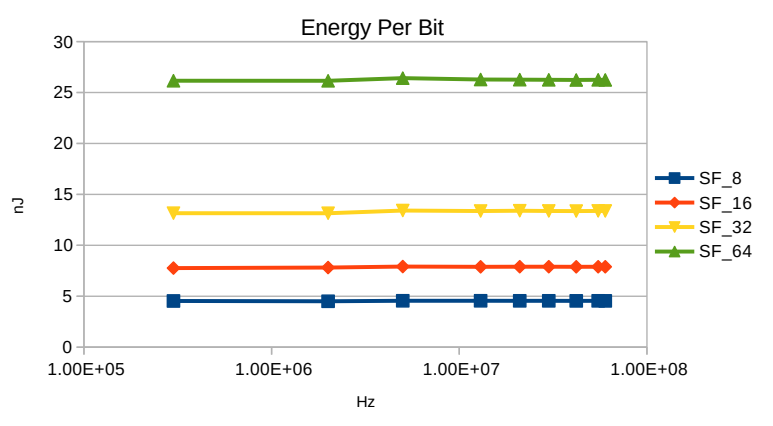

Figure 7: Transmitter power consumption vs frequency for each IEEE 802.15.6 spreading factor (SF)

factor approximately doubles the packet length. For spreading factors of $8,16,32$ and 64 chips per bit, the transmitter power requirements are approximately $4.5 \mathrm{~nJ}, 7.9 \mathrm{~nJ}, 13.4 \mathrm{~nJ}$ and 26.3nJ per bit respectively. The IEEE 802.15.6 transmit spectral mask will require further power, however the prototype transmitter achieves this using analogue filtering, offering an improvement in power consumption over the approach of digital filtering followed by a digital to analogue converter [5].

\subsection{Channel attenuation}

Figure 8 plots the channel attenuation at each transmission frequency and electrode separation. Also included are the results with the phantom solution replaced with air. The results are consistent with those produced by Choo et al. [2] and Lucev et al. [4] in that the channel exhibits high pass characteristics initially, although no substantial low pass effect was observed above $45 \mathrm{MHz}$. As the communications channel relies on capacitive coupling for the ground path, the experiment environment will have significant influence on the results.

Looking at the air coupling results, it can be seen that generally, RF coupling of the signal increases with frequency. Within the environmental conditions of this experiment, the largest difference between RF coupled reception and capacitively coupled reception occurs at $21 \mathrm{MHz}$.

For comparison with the RF based approach presented in IEEE 802.15.6 section 9, Wang et al. demonstrate that over a similar distance, ultra wideband (UWB) communications show losses closer to $60 \mathrm{~dB}[8]$.

As shown in Table 2 the difference between attenuation for 


\begin{tabular}{cccc}
\hline & $\begin{array}{c}11.25 \mathrm{~cm} \\
\text { Attenuation } \\
(\mathrm{dB})\end{array}$ & $\begin{array}{c}22.5 \mathrm{~cm} \\
\text { Attenuation } \\
(\mathrm{dB})\end{array}$ & $\begin{array}{c}\text { Attenuation } \\
\text { Difference } \\
(\mathrm{dB})\end{array}$ \\
\hline \hline $300 \mathrm{kHz}$ & 46.5 & 46.8 & 0.3 \\
$2 \mathrm{MHz}$ & 29.6 & 29.5 & -0.2 \\
$5 \mathrm{MHz}$ & 21.9 & 22.3 & 0.4 \\
$13 \mathrm{MHz}$ & 10.1 & 11.9 & 1.8 \\
$21 \mathrm{MHz}$ & 9.8 & 11.4 & 1.6 \\
$30 \mathrm{MHz}$ & 16.6 & 18.4 & 1.8 \\
$42 \mathrm{MHz}$ & 12.8 & 13.6 & 0.8 \\
$55 \mathrm{MHz}$ & 12.5 & 11.0 & -1.5 \\
$60 \mathrm{MHz}$ & 10.8 & 12.9 & 2.1 \\
\hline
\end{tabular}

Table 2: Channel attenuation in phantom solution for each transmission frequency. Attenuation difference shown is the difference in attenuation for $d=11.25 \mathrm{~cm}$ and $d=22.5 \mathrm{~cm}$.

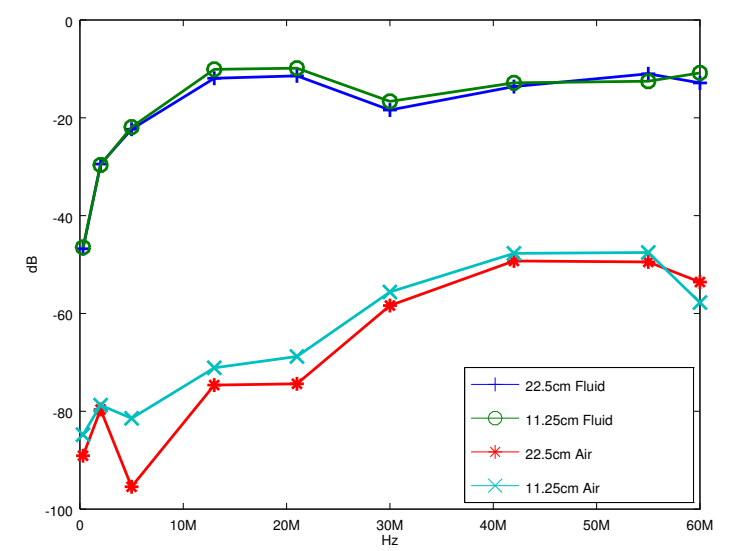

Figure 8: Channel attenuation in phantom solution and in air for $d=11.25 \mathrm{~cm}$ and $d=22.5 \mathrm{~cm}$.

$d=11.25 \mathrm{~cm}$ and $d=22.5 \mathrm{~cm}$ averages $1.7 \mathrm{~dB}$ from $13 \mathrm{MHz}$ to $30 \mathrm{MHz}$. At higher frequencies, increased air coupling makes it difficult to isolate the effect of the increased spacing. This experiment does not take into account variations in environment, which may have more significant impact on attenuation than electrode separation. The relationship between environment and channel attenuation will be further investigated in future experiments.

Figures 9 and 10 show the spectrum of the transmitted signal (in green, normalised) and the received signal (in blue) at $30 \mathrm{MHz}$, with $d=22.5 \mathrm{~cm}$ and spreading factor 8 though the phantom solution and a male test subject's arm respectively. While there is a $6 \mathrm{~dB}$ difference in attenuation at the peak frequency of $30 \mathrm{MHz}$ between the two mediums, the channel frequency characteristics are similar, demonstrating the feasibility of the use of a phantom solution to model the human body channel in a controlled environment.

The experimental setup allows complete contact between the electrodes and the phantom solution, while contact with the subject's arm is often degraded. The difference in attenuation is consistent with variations in attenuation due to electrode contact degradation seen by Lucev et al. [4]. The rela-

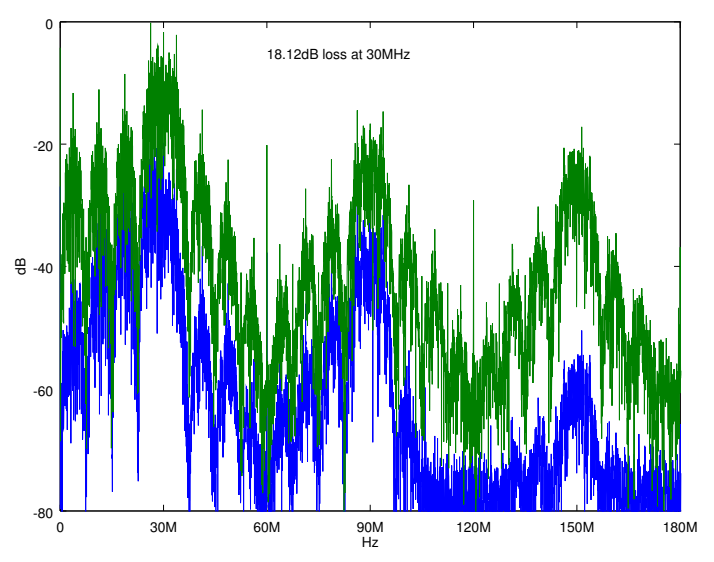

Figure 9: Spectrum plot of received signal (blue) and reference signal (green) at $30 \mathrm{MHz}$ through the phantom solution with $d=22.5 \mathrm{~cm}$.

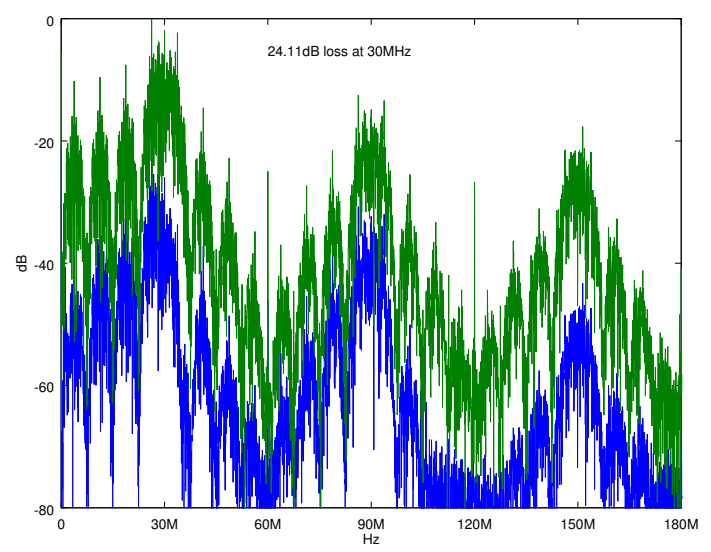

Figure 10: Spectrum plot of received signal (blue) and reference signal (green) at $30 \mathrm{MHz}$ through a male test subject's arm with $d=22.5 \mathrm{~cm}$.

tionship between channel attenuation and electrode contact will be further investigated using the prototype transceiver in real world applications.

Figure 11 is a time-domain plot of the transmitted signal (in green) and the received signal (in blue) at $21 \mathrm{MHz}$ with a spreading factor of 8 . The effect of the capacitive coupling can be most easily observed at each phase transition where the received signal decays towards zero during the two simultaneous chips. It is here that incorrect hard decision results at the output of the comparator in Figure 5 are most likely.

One of the consequences of sampling the data with a fixed clock frequency occurs when there is drift in clock frequency. In such cases, the receiver may for example move from sampling at $180^{\circ}$ to $90^{\circ}$ to $0^{\circ}$ to $270^{\circ}$. In this case the receiver must "wrap" to the previously sampled chip. The sensitivity to clock jitter is then proportional to the size of the input 


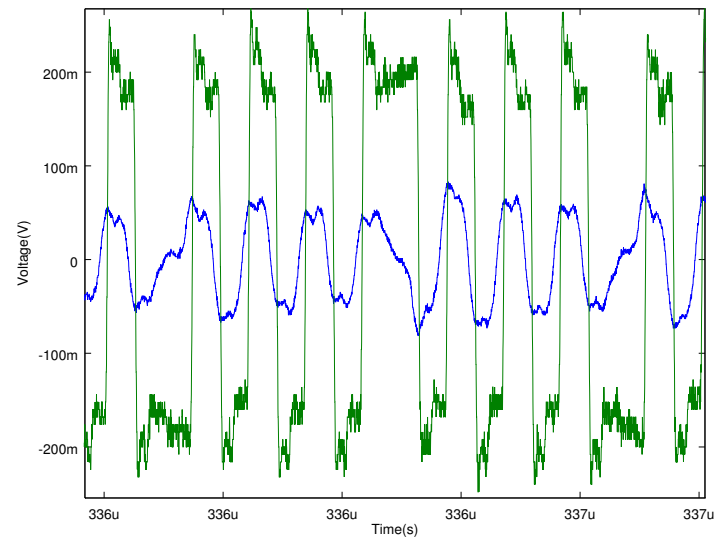

Figure 11: Time domain plot of received signal (blue) and reference signal (green) at $21 \mathrm{MHz}$, spreading factor $\mathbf{8}$ with $d=22.5 \mathrm{~cm}$

sample buffer. The sample buffer has been set to 64 in the prototype receiver design.

The presence of noise, combined with the transient response of the input filter would occasionally cause the receiver data recovery stage to incorrectly "wrap", leading to an extra chip detected and a corresponding loss of synchronisation. This was the case with the $5 \mathrm{MHz}$ BER measurements presented below.

The lower incidence of phase changes with higher spreading factors helps to alleviate this. With higher spreading factors, the channel tends to stabilise on the current phase, allowing the receiver to more easily detect the phase information, in the presence of noise.

\subsection{Bit error rate performance}

With a maximum sample duration of $2 \mathrm{~ms}$ for the oscilloscope, it was not possible to demodulate packets at $300 \mathrm{kHz}$ or $2 \mathrm{MHz}$ with a spreading factor of 64 . For all other packet types, Figures 12 and 13 show the raw and demodulated bit error rates respectively. Note that for all spreading factors, at $5 \mathrm{MHz}$, the receiver was unable to correctly synchronise. Similarly at a spreading factor of 8 synchronisation was not achieved at 21 and $42 \mathrm{MHz}$. For all other frequencies and spreading factors the receiver was able to synchronise with correct demodulation except at $42 \mathrm{MHz}$, spreading factor 16 .

The number of chips captured during the $2 \mathrm{~ms}$ sampling interval varied from 4096 chips at $300 \mathrm{MHz}$ to 233474 chips at $60 \mathrm{MHz}$. Incorrect chip detection around the phase transitions was the largest contributing factor to the raw BER. This can be seen in 12 where the BER improves for larger spreading factors. The compromise between energy per bit requirements and BER performance may be seen by comparing figures 7 and 12 .

Achieving reliable synchronisation at the intended operating frequency requires customisation of the analogue front end filter to the transmission frequency. Further work to improve

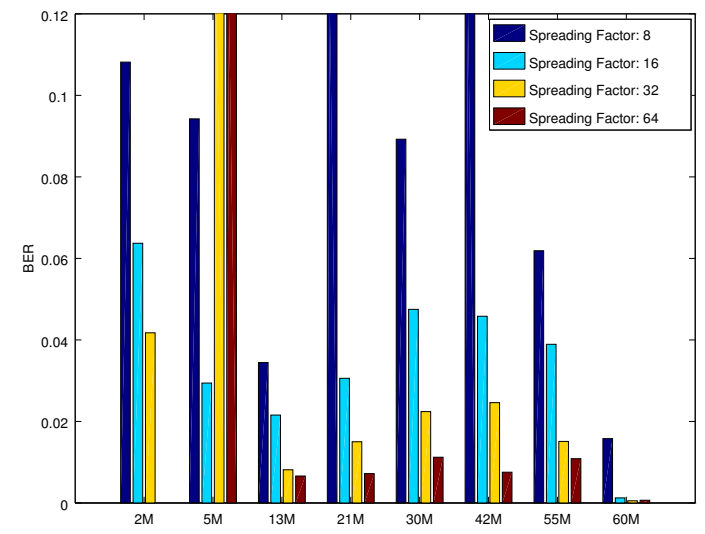

Figure 12: Plot of BER at the receiver before after hard decision detection.

the receiver's data recovery stage will be investigated.

The use of Walsh coding provides effective error correction over the channel under consistent environmental conditions. For example, at a spreading factor of 8 at $30 \mathrm{MHz}$, the receiver was able to correct 5940 hard decision bit errors and correctly reconstruct the packet. A comparison of Figures 12 and 13 demonstrates the effectiveness of the error correction scheme. The Walsh coding algorithm presented in IEEE 802.15.6 allows for a low complexity receiver through the use of LUTs [7], reducing the logic requirements of the receiver. The 64 bit hamming distance LUT implemented in the prototype receiver requires 176 bit LUTs. Future testing of environmental fading found in real world applications will be undertaken in order to characterise the performance of the error correction scheme.

Figure 12 demonstrates improvements in raw BER with frequency. While this might suggest that higher frequencies will be more attractive for capacitively coupled human body communications, the increase in air coupling shown in Figure 8 suggests that RF propagation is becoming more dominant at high frequencies.

\section{CONCLUSION}

This experiment has shown that reliable communications using capacitive coupling with the IEEE 802.15.6 protocol may be achieved using low cost FPGA technology combined with simple analogue interfacing. With channel attenuation as low as $11.4 \mathrm{~dB}$ over $22.5 \mathrm{~cm}$, capacitive coupling offers significantly lower attenuation than an RF based approach. As seen in this study, transmitter power consumption may be as low as $4.5 \mathrm{~nJ}$ per bit, using FPGA technology. Development of a portable prototype transceiver will allow for rapid channel attenuation measurement in variety of environments, including those typically experienced by an end user. This information will be used in future assessment of the efficacy of the modulation scheme proposed by IEEE 802.15.6.

\section{REFERENCES}




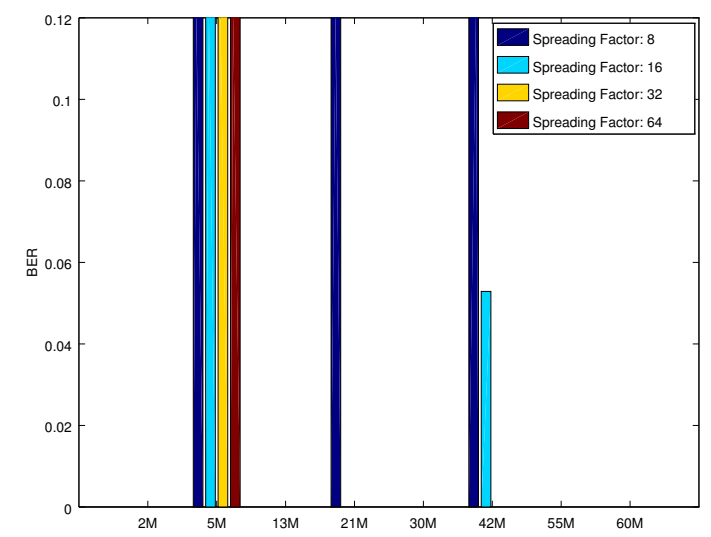

Figure 13: Plot of BER demodulation by the prototype receiver. Loss of synchronisation is the cause of the large BER seen at $5 \mathrm{MHz}$.

[1] IEEE Standard for Local and metropolitan area networks - Part 15.6: Wireless Body Area Networks. IEEE Std 802.15.6-2012, pages 1-271, Feb. 2012.

[2] N. Cho, J. Yoo, S.-J. Song, J. Lee, S. Jeon, and H.-J. Yoo. The Human Body Characteristics as a Signal Transmission Medium for Intrabody Communication. Microwave Theory and Techniques, IEEE Transactions on, 55(5):1080-1086, May 2007.

[3] M. Hagmann, R. Levin, L. Calloway, A. Osborn, and K. Foster. Muscle-equivalent phantom materials for 10-100 MHz. Microwave Theory and Techniques, IEEE Transactions on, 40(4):760-762, 1992.

[4] Z. Lucev, I. Krois, and M. Cifrek. A Capacitive Intrabody Communication Channel from $100 \mathrm{kHz}$ to $100 \mathrm{MHz}$. Instrumentation and Measurement, IEEE Transactions on, 61(12):3280-3289, Dec. 2012.

[5] G. Makkena, K. Abhilash, and M. Srinivas. Gaussian filter approximation using Levin's transformation for implementation in analog domain. In Microelectronics and Electronics (PrimeAsia), 2013 IEEE Asia Pacific Conference on Postgraduate Research in, pages 204-207, Dec. 2013.

[6] Nick Sawyer. XAPP225: Data to Clock Phase Alignment, Feb. 2009.

[7] V. Sklyarov and I. Skliarova. Digital Hamming weight and distance analyzers for binary vectors and matrices.

[8] J. Wang and Q. Wang. Body Area Communications: Channel Modeling, Communication Systems, and EMC. Wiley-IEEE Press, 2012.

[9] Xilinx Inc. UG733: Xilinx Power Tools Tutorial, Mar. 2010. 